\title{
Inflammatory Bowel Disease and Clostridium difficile Infection: A Report from a Tertiary Care Center of North India
}

Chetana Vaishnavi", Rakesh Kochhar

Department of Gastroenterology, Postgraduate Institute of Medical Education and Research, Chandigarh, India

*Corresponding author: Chetana Vaishnavi, Department of Gastroenterology, Postgraduate Institute of Medical Education and Research, Chandigarh, India Tel: 91-172-2756609; E-mail: c.vaishnavi@rediffmail.com

Received date: May 15, 2017; Accepted date: May 18, 2017; Published date: May 25, 2017

Copyright: ( 2017 Vaishnavi C. This is an open access article distributed under the terms of the Creative Commons Attribution License, which permits unrestricted use, distribution, and reproduction in any medium, provided the original author and source are credited

\begin{abstract}
Clostridium difficile infection (CDI) is reported to be frequently associated with inflammatory bowel disease (IBD). Because of the common characteristics of the clinical appearance of CDI and IBD the diagnosis and treatment of the same becomes complicated. We retrospectively investigated the prevalence of CDI in patients with IBD and analyzed their demographic and clinical profile. During analysis, the IBD patients $(n=721 ; M: F=1.10: 1)$ comprised of test group and an equal number of gender-matched patients with no indication of IBD was included as non-IBD controls. The demographic and clinical data as well as fecal $C$. difficile toxin status of all the patients were retrieved from our meticulously maintained laboratory records. The number of $C$. difficile positivity was more in the non-IBD group $(20 \%)$ compared to IBD patients $(16 \%)$ with non-significant difference $(p<0.064)$. The patients in non-IBD group were relatively older $(p<0.001)$ compared to those in IBD group. The duration of diarrhea in the IBD group was significantly longer $(p<0.001)$ compared to the non-IBD group, but no significant difference $(p>0.063)$ in the frequency of diarrhea between the two groups was observed. Blood in stool and abdominal pain symptoms were found to be significantly higher $(p<0.001)$ among the IBD group whereas watery diarrhea and fever were significantly linked $(p<0.001)$ with non-IBD group. Significant improvement was seen in most of the parameters during the time of follow-up. Though the prevalence of CDI in both IBD and non-IBD groups were almost comparable, clinical symptom and age of presentation varied in them.
\end{abstract}

Keywords: Abdominal pain; Clostridium difficile; Diarrhea; Followup studies; Humans; Inflammatory bowel disease; Prevalence

\section{Introduction}

Clostridium difficile is notorious in causing infectious colitis usually in the elderly or hospitalized patients, or those with exposure to antibiotics [1]. However, recently there is a significant rise in $C$. difficile infection (CDI) incidence in the general population involving younger age group, and those patients who have undergone transplant or are immunocompromised [2]. An increase in the community prevalence of CDI is also being reported $[3,4]$.

Inflammatory bowel diseases (IBD) broadly comprise of ulcerative colitis (UC) and Crohn's disease (CD) and such patients have been identified to be at increased risk of acquiring CDI in relation to the general population $[5,6]$ with greater proportion associated with hospitalization, surgery, as well as mortality when compared to nonIBD patients with CDI $[7,8]$. The incidence of CDI among IBD patients has shown an increase of 2-3 folds [9] particularly including younger age-group, community acquisition and lack of antibiotic exposure [10]. Patients with IBD also experience worse outcomes [11] and high risk of CDI recurrence [12]. However, the reason for increased carriage and CDI risk in IBD patients is not quite clear.

C. difficile infection in India is different in the context of it being less severe. Data available of CDI in IBD patients in India is sparse [13]. A significant interest regarding the association of CDI with IBD is growing amongst workers $[14,15]$ warranting increased vigilance regarding $\mathrm{CDI}$ in IBD patients. We receive fecal samples from patients suspected for CDI from different wards of the hospital, along with pre- printed pro-forma duly filled up by the treating clinicians. This paper pro-forma is specially designed to note down all information pertaining to patients suspected for CDI and is maintained in our laboratory where they are kept in order and serially bound in a collection each comprising of 200 consequent pro-forma for future analysis. From this meticulously maintained laboratory records, we retrospectively investigated the prevalence of CDI in patients with IBD and analysed their clinical and demographic profile in relation to gender-matched non-IBD patients reporting during the same study period.

\section{Patients and Methods}

This work was carried out in the Microbiology Division, Department of Gastroenterology, Post Graduate Institute of Medical Education and Research, Chandigarh, India, which is a 2100 bedded tertiary care teaching hospital where patients from different states of North India receive treatment. The period of data collection was from October 2009 to May 2016. Patients suspected of CDI from both in and out-patient services as a matter of routine care formed the basis of investigation. Baseline and follow-up information of patients were obtained from laboratory records. Informed consent could not be taken as this is a retrospective analysis. The Institute Research Ethics Committee which operates according to the Helsinki Declaration approved the investigation.

All consecutive patients excluding pregnant women and children under 2 years of age reporting to the tertiary care hospital during the study period were enrolled for analyses. Patients whose data could not be completely acquired were removed from the analysis. A total of 721 IBD patients (711 UC; $10 \mathrm{CD}$ ) with an acute flare of the disease were 
Page 2 of 6

included in the study. Diagnosis of IBD was done by the clinicians based on the patient's signs and symptoms and/or evaluation of endoscopic, histopathological and radiological information. An equal number of gender-matched patients $(\mathrm{n}=721)$ referred for CDI but without any known history of IBD was included in the non-IBD group. Thus a total of 1442 patients who primarily provided fecal samples for CDI were investigated. Fecal samples for CDI investigation was also provided by 177 UC patients and 48 non-IBD patients on subsequent visits. Though fecal samples were received on two or three occasions in the follow-up cases, only the baseline and the last follow-up samples were analyzed. In the present study the follow-up of patients both in the IBD and in the non-IBD groups were carried out for a period of six years.

All submitted fecal specimens received in sterile containers are processed immediately and $C$. difficile toxins $\mathrm{A}$ and $\mathrm{B}$ detected using ELISA kits. The laboratory records were reviewed for patient demographics, clinical presentation, medical history, etc. All prescriptions for antibiotics and other drugs were also taken into consideration.

\section{Statistical analysis}

The whole data were entered into excel master sheet and analyzed by using SPSS 22.0 (IBM Corporation and other(s), 1989, 2013). Continuous variables like age of the patients and duration and frequencies of diarrhea were compared using Student's t-test. Other categorical variables were analyzed using Chi-square test. Follow-up data were analyzed using McNemar-Bowker test separately for each of the clinical symptoms in both the groups.

\section{Results}

\section{IBD group}

Of the 721 IBD patients analyzed in the study, 393 were males and 328 were females (1.10:1). The age range of the IBD patients was 6 to 86 years (mean age 37.5 years). There were $343(47.6 \%)$ hospitalized patients, $362(50.2 \%)$ outpatients and $16(2.2 \%)$ patients with hospitalization status unknown. Predominant clinical symptoms present in patients with IBD were watery diarrhea in $32.7 \%$, abdominal pain in $50 \%$ and fever in $15.5 \%$. Bloody diarrhea was found in 270 (37.4\%) of the patients indicating active disease, whereas 451 (62.6\%) patients were non-bleeding (inactive disease). Mucus was observed in only $30 \%$ of all the samples. Antibiotics were received by $35.9 \%$ patients, with $49 \%$ of them receiving multiple antibiotics. C. difficile toxin was positive in $16 \%$ of IBD patients. Usage of antibiotics was not significant $(\mathrm{p}=0.304)$ in relation to $C$. difficile positivity. Steroids were received by $13.2 \%$ of the patients and proton pump inhibitors (PPI) by $5.1 \%$.

\section{Non-IBD group}

In the gender-matched, non-IBD patients $(n=721)$ the age ranged from 3 to 90 years (mean age 45.1 years). There were 652 (90.4\%) hospitalized patients, 52 (7.2\%) outpatients and 17 (2.4\%) patients with hospitalization status unknown. Predominant clinical symptoms observed were watery diarrhea in $99.7 \%$, pain abdomen in $40 \%$ and fever in $43 \%$ of the patients in the non-IBD group. Blood in stool was present in only $5.7 \%$ though mucus was seen in $41.5 \%$ of all the samples. C. difficile toxin was positive in $19.8 \%$ of non-IBD patients. Usage of antibiotics was not significant $(\mathrm{p}=0.238)$ in relation to $C$. difficile positivity. Fifty three (7.3\%) of the non-IBD patients used PPI.

\section{Comparative analysis of IBD group versus non-IBD group}

Table 1 depicts comparative analysis of various parameters in the IBD and the non-IBD groups. There was no significant difference ( $p$ value $=1$; Chi square $=0$ ) in the proportion of males and females among the IBD and the non-IBD groups because of gender-matched population included in the study. Age was found to be significantly different $(\mathrm{p}<0.001)$ between the IBD and the non-IBD groups with patients in the non-IBD group being relatively older (mean age $45.15 \pm$ SD 16.7 years) as compared to the IBD group (mean age $37.59 \pm \mathrm{SD}$ 13.2 years). Though the number of $C$. difficile toxin positivity was more in the non-IBD group compared to the IBD group, there was no significant difference $(\mathrm{p}=0.064)$ between the two groups. On comparison of the frequency and duration of diarrhea between both the groups, the mean frequency was not found to be significantly different $(\mathrm{p}<0.05)$, but the mean duration was significantly longer $(\mathrm{p}<0.001)$ in the IBD patients (mean 225 days) in comparison to the non-IBD patients (mean 38 days).

\begin{tabular}{|c|c|c|c|}
\hline Variables & $\begin{array}{l}\text { IBD group } \\
n=721 \text { (\%) }\end{array}$ & $\begin{array}{l}\text { Non-IBD group } \\
n=721(\%)\end{array}$ & $P$ values \\
\hline Male:Female & $393: 328$ & $393: 328$ & 1 \\
\hline Age (Mean \pm SD) & $37.53 \pm 13.20$ & $45.15 \pm 16.72$ & $<0.001$ \\
\hline C. difficile positive & $116(16.09)$ & $143(19.83)$ & 0.064 \\
\hline \multicolumn{4}{|l|}{ Clinical symptoms } \\
\hline Watery diarrhea & $236(32.73)$ & 719 (99.72) & $<0.001$ \\
\hline Abdominal pain & $363(50.35)$ & $288(39.94)$ & $<0.001$ \\
\hline Fever & $112(15.53)$ & $311(43.14)$ & $<0.001$ \\
\hline \multicolumn{4}{|l|}{ Diarrhea attributes } \\
\hline Frequency/day Mean \pm SD & $6.56 \pm 3.79$ & $6.96 \pm 4.02$ & 0.063 \\
\hline
\end{tabular}


Citation: Vaishnavi C, Kochhar R (2017) Inflammatory Bowel Disease and Clostridium difficile Infection: A Report from a Tertiary Care Center of North India. J Gen Pract (Los Angel) 5: 310. doi:10.4172/2329-9126.1000310

Page 3 of 6

\begin{tabular}{|l|l|l|l|}
\hline Duration(days) & 224.6 & 38 & $<0.001$ \\
\hline Blood in stool & $270(37.45)$ & $41(5.68)$ & $<0.001$ \\
\hline Presence of mucus & $218(30.24)$ & $299(41.47)$ & $<0.001$ \\
\hline Antibiotics usage & \multicolumn{3}{|l|}{} \\
\hline Nil & $462(64.08)$ & $48(6.66)$ & $<0.001$ \\
\hline Single & $131(18.17)$ & $283(39.25)$ & $<0.001$ \\
\hline Multiple & $128(17.75)$ & $390(54.09)$ & $<0.001$ \\
\hline
\end{tabular}

Table 1: Comparative analysis of various parameters in the IBD and the non-IBD groups.

When the use of antibiotics amongst both the IBD and the nonIBD groups was analyzed, a significant higher association $(\mathrm{p}<0.001)$ in the usage of antibiotics in non-IBD group compared to the IBD group was observed. However, the usage was non-significant (IBD $\mathrm{p}=0.304$; non-IBD $\mathrm{p}=0.238$ ) in relation to $C$. difficile positivity in both the groups. A significant difference $(\mathrm{p}=0.014)$ in the usage of multiple antibiotics compared to single or no antibiotics in relation to $C$. difficile positivity was also observed. Table 2 shows $C$. difficile toxin outcome in relation to antibiotic use in general and Table 3 shows $C$. difficile toxin status in relation to antibiotic use in IBD and non-IBD groups.

There was no significant difference $(\mathrm{p}>0.05)$ in the use of PPI in the IBD group compared to the non-IBD group.

\begin{tabular}{|l|l|l|l|}
\hline Antibiotics & C. difficile positive $\mathbf{n}(\%)$ & C. difficile negative $\mathbf{n}(\%)$ & Total \\
\hline Nil & $75(28.96)$ & $435(36.77)$ & $510(35.37)$ \\
\hline Single & $72(27.8)$ & $342(28.91)$ & $414(28.71)$ \\
\hline Multiple & $112(43.24)$ & $406(34.32)$ & $518(35.92)$ \\
\hline Total & $259(100)$ & $1183(100)$ & $1442(100)$ \\
\hline
\end{tabular}

Table 2: $C$. difficile toxin outcome in relation to antibiotic usage in general.

\begin{tabular}{|c|c|c|c|c|c|c|c|c|}
\hline \multirow[t]{2}{*}{ Antibiotics } & \multicolumn{4}{|l|}{ IBD } & \multicolumn{4}{|l|}{ Non-IBD } \\
\hline & $\begin{array}{l}\text { C. difficile positive } \\
\mathrm{n}(\%)\end{array}$ & $\begin{array}{l}\text { C. difficile negative } \\
\mathrm{n}(\%)\end{array}$ & $\begin{array}{l}\text { Total } \\
\mathrm{n}(\%)\end{array}$ & $P$ value & $\begin{array}{l}\text { C. difficile positive } \\
\mathrm{n}(\%)\end{array}$ & $\begin{array}{l}\text { C. difficile negative } \\
\mathrm{n}(\%)\end{array}$ & $\begin{array}{l}\text { Total } \\
\mathrm{n}(\%)\end{array}$ & $P$ value \\
\hline Nil & $68(58.62)$ & $394(65.12)$ & $462(64.08)$ & 0.304 & $7(4.9)$ & $41(7.09)$ & $48(6.66)$ & 0.238 \\
\hline Single & $22(18.97)$ & 109 (18.02) & $131(18.17)$ & 0.304 & $50(34.97)$ & $233(40.31)$ & $283(39.25)$ & 0.238 \\
\hline Multiple & $26(22.41)$ & $102(16.86)$ & $128(17.75)$ & 0.304 & $86(60.14)$ & $304(52.6)$ & $390(54.09)$ & 0.238 \\
\hline
\end{tabular}

Table 3: Antibiotics in relation to $C$. difficile toxin status in individual groups.

\section{Follow-up groups}

There were 177 follow-up patients in the IBD group (age range 16-68 years) and 48 in the non-IBD group (age range $4-76$ years). Table 4 shows a comparative analysis of various parameters between the IBD and the non-IBD groups at follow-up. Among the follow-up groups, there was a significant difference $(\mathrm{p}<0.001)$ between the mean duration of diarrhea in the IBD group as compared to the non-IBD group. In $C$. difficile positive group there was a significant difference $(\mathrm{p}=0.026)$ in the mean duration of diarrhea between the IBD versus the non-IBD groups. Similarly among $C$. difficile negative group also there was a significant difference $(\mathrm{p}<0.001)$ in the mean duration of diarrhea between both the groups. However, in both the IBD and the non-IBD groups upon individual analysis there was no significant difference
( $>>0.05)$ in the mean duration of diarrhea between $C$. difficile positive versus $C$. difficile negative groups. In the IBD group there was a significant decrease $(p<0.001)$ in the number of watery diarrhea at the time of follow-up compared to the base line data. Fever was found to be significantly present $(\mathrm{p}<0.001)$ in the IBD group at the time of primary reporting compared to that at the time of follow-up. Significant decrease $(\mathrm{p}<0.001)$ in the number of $\mathrm{C}$. difficile negative IBD patients with fever symptom at the time of follow-up was observed compared to that at the time of admission. Among the $C$. difficile negative IBD group patients there was a significant change $(\mathrm{p}<0.001)$ in the number of antibiotics used at the time of preliminary reporting compared to that at the time of follow-up. 


\begin{tabular}{|c|c|c|c|}
\hline Variables & $\begin{array}{l}\text { IBD } \\
n=177(\%)\end{array}$ & $\begin{array}{l}\text { Non-IBD } \\
(n=48)\end{array}$ & P values ${ }^{*}$ \\
\hline Male: Female & $94: 83$ & $31: 17: 00$ & 0.15 \\
\hline Age (Mean \pm SD) & $36.44 \pm 11.90$ & $41.38 \pm 19.52$ & $0.03^{*}$ \\
\hline C. difficile positive & $34(19.2)$ & $12(25.0)$ & 0.38 \\
\hline \multicolumn{4}{|l|}{ Clinical symptoms } \\
\hline Watery diarrhea & $57(32.2)$ & 45 (93.7) & $0.00^{*}$ \\
\hline Abdominal pain & 77 (43.5) & $16(33.3)$ & 0.2 \\
\hline Fever & $26(14.7)$ & $18(37.5)$ & $0.00^{*}$ \\
\hline \multicolumn{4}{|l|}{ Diarrhea attributes } \\
\hline Duration (days) & $1-1095$ & Jan-60 & $<0.001$ \\
\hline Blood in stool & $45(25.4)$ & $7(14.6)$ & 0.11 \\
\hline Presence of mucus & $48(27.1)$ & $16(33.3)$ & 0.39 \\
\hline Antibiotic usage & $107(60.4)$ & $46(95.8)$ & $0.00^{*}$ \\
\hline
\end{tabular}

Table 4: Follow-up patients: Comparative analysis of various parameters in the IBD and the non-IBD groups.

\section{Discussion}

The risk of $C$. difficile acquisition with IBD patients has increased in frequency and severity [10]. The carriage of toxin producing $C$. difficile has been more often found in IBD patients in comparison to the general population $[16,17]$. Evidence has been established that IBD patients have gut flora which is different from patients without IBD [18], predisposing to colonization with $C$. difficile and other pathogens. This is particularly true because of their innate immune deficiencies, use of antibiotics and immunosuppressives for a long period of time as well as frequent hospitalizations [19]. In the present study 95 (13.2\%) IBD patients were on steroids and at least $212(29 \%)$ were hospitalized for treatment. Uncommon features like recurrent bloody stools, younger age-group patients and no previous hospital contact may be found in IBD patients with CDI [20,21]. In the present study a very large number of patients in the IBD group had bloody stools compared to the non-IBD group and the patients were also significantly younger. However, $C$. diffiicile toxin positivity was almost similar in both the IBD and the non-IBD groups, contrary to expectations. Ott et al. [22] also reported low risk of CDI in hospitalized patients with IBD in a tertiary referral center in Germany. Low prevalence of $C$. difficile in IBD patients was also reported by Masclee et al. [23] indicating that $C$. difficile does not commonly elicit IBD flare-ups in The Netherlands. In an earlier preliminary investigation we also observed that there was insignificant risk of CDI in IBD cases [13] probably as the hyper virulent strains present in other geographical region has not been detected here so far [24]. Moreover patients in the non-IBD group comprised largely of hospitalized patients suspected of CDI because of diarrhea.

An episode of CDI is characterized by watery diarrhea with abdominal cramps and fever [13]. The similar presentation of abdominal pain and diarrhea, makes diagnosis of CDI in IBD patients difficult. A higher prevalence of asymptomatic CDI is seen in IBD. Watery diarrhea was present in less than one-third of the IBD patients to that present in the non-IBD group. Fever was also similarly present in a little more than one-third of the IBD patients to that present in the non-IBD group. Abdominal pain was However, found to be more frequent in presentation in the IBD group probably because of the inflammatory nature of the disease. Presence of mucus in stool is also a distinguishing aspect of $C$. difficile diarrhea [25]. However, in our IBD patients, the number of stool samples with mucus was much less compared to that of the non-IBD group. The only explanation in this context could be that lesser mucus was produced as the patients were under treatment or because mucus was probably camouflaged due to the presence of blood in stool in many of the IBD patients.

Apart from the usually encountered risk factors for CDI [26] diverse studies have found that IBD patients are at higher risk of developing CDI with varying risk amongst diverse patient subsets. Libby et al. [27] reported that $76 \%$ of CDI in IBD patients signify community acquired infections in contrast to those without IBD where the majority portion of CDI are hospital acquired as corroborated by others workers [28,29]. In our study there were $436 \mathrm{IBD}$ patients treated as outpatients and of these $66(15 \%)$ were positive for CDI. Similarly a rate close to $60 \%$ of antibiotic exposure in CDI affected IBD patients has been reported [20] implying thereby that the reduced diversity of microbiota in the IBD patients may be a predisposing factor for $C$. difficile colonization [30]. However, up to $40 \%$ of the IBD patients do not have recognized antibiotic exposure preceding CDI presentation [31]. In the present study also there was a significantly lesser use of antibiotic in the IBD patients compared to the non-IBD group.

It has been reported that CDI can trigger flares in IBD patients and cause further deterioration of the disease [32,33] regardless of CDI treatment. There are several reports of the incidence of CDI in IBD patients establishing that these patients have between two and seven times higher incidence of CDI than those without IBD [6,20,34,35]. When a patient with IBD relapses, detection of $C$. difficile or its toxin in fecal samples confirms the part played by the organism in the inflammatory process of the colon. Hookman et al. [2] found that 
fulminant colitis is more often reported at times of $C$. difficile outbreaks in IBD patients with increased mortality than in those with no primary IBD.

Patients with UC are more vulnerable to CDI and have added severe outcomes compared to CD patients [20-34]. In a retrospective study Rodemann et al. [6] reported in a tertiary care hospital that the CDI occurrence in CD doubled and that in UC tripled over a 7-year period (1998-2004). In the present investigation, there were 113 UC and 3 CD patients with CDI. Hughes et al. [36] reported that UC patients present with lower IgA levels to $C$. difficile toxins in comparison to $\mathrm{CD}$ patients, and that IBD patients with preceding CDI did not have any increased antitoxin IgG. The limitation of this study was that CD cases were very few and therefore, CDI outcomes in CD could not be properly assessed.

The limitation of this study is that it is a retrospective analysis. However, it may be reiterated that records are well maintained in duly filled up pro-forma specially designed to note down all information pertaining to patients suspected for CDI for probable future analysis. The strength of this study is that follow-up patients were available for both IBD and non-IBD groups taken from a similar setting. Significant improvement was seen in all the parameters upon treatment and follow-up. However, upon comparison of various parameters between IBD and non-IBD follow-up groups, it was observed that there was a highly significant difference in variables like age, watery diarrhea, fever and the use of antibiotics between both the groups. This differentiates and highlights the factors associated with clinical suspicion of both IBD and non-IBD cases.

Clinicians should be cautious about the chances of CDI in patients who have an exacerbation of IBD. At times the IBD flare cannot be differentiated from CDI requiring a high degree of clinical suspicion and vouching for early stool testing for toxin assay [37]. When CDI in IBD are established primarily within two days of hospital admission it suggests that a good number of the infection was acquired before admission. CDI should, therefore, be suspected in differential diagnosis for intractable IBD patients, because many such patients need not present with a history of antibiotic exposure or hospital admission and may largely be receiving outpatient treatment. Absence of antibiotic exposure should not decrease the suspicion of CDI [38] in IBD patients. Even when diarrhea is not present, presence of other clinical symptoms and laboratory findings indicate a potential infectious condition, ruling out $C$. difficile would be a practical measure. Careful surveillance for CDI among all IBD patients exhibiting a flare of the disease should be done for early identification and aggressive treatment for reducing the morbidity of CDI among them.

\section{References}

1. McDonald LC, Owings M, Jernigan DB (2006) Clostridium difficile infection in patients discharged from US short-stay hospitals, 1996-2003. Emerg Infect Dis 12: 409-415.

2. Hookman P, Barkin JS (2009) Clostridium difficile associated infection, diarrhea and colitis. World J Gastroenterol 15: 1554-1580.

3. Dial S, Delaney JA, Schneider V, Suissa S (2006) Proton pump inhibitor use and risk of community-acquired Clostridium difficile-associated disease defined by prescription for oral vancomycin therapy. CMAJ 175: 745-748.

4. Sammons JS, Toltzis P (2013) Recent trends in the epidemiology and treatment of Clostridium difficile infection in children. Curr Opin Pediatr 25: 116-121.
5. Vaishnavi C, Kochhar R, Bhasin, DK, Thennarasu K, Singh K (2003) Simultaneous assay for Clostridium difficile and fecal lactoferrin in ulcerative colitis. Trop Gastroenterol 24: 13-16.

6. Rodemann JF, Dubberke ER, Reske KA, Seo DH, Stone CD (2007) Incidence of Clostridium difficile infection in inflammatory bowel disease. Clin Gastroenterol Hepatol 5: 339-344.

7. Ananthakrishnan AN, McGinley EL, Binion DG (2008) Excess hospitalisation burden associated with Clostridium difficile in patients with inflammatory bowel disease. Gut 57: 205-210.

8. Tang YM, Stone CD (2008) Clostridium difficile infection in inflammatory bowel disease: challenges in diagnosis and treatment. Clin J Gastroenterol 10: 112-113.

9. Schneeweiss S, Korzenik J, Solomon DH, Canning C, Lee J, et al. (2009) Infliximab and other immunomodulating drugs in patients with inflammatory bowel disease and the risk of serious bacterial infections. Alimentary Pharma Therap 30: 253-264.

10. Berg AM, Kelly CP, Farraye FA (2013) Clostridium difficile infection in the inflammatory bowel disease patient. Inflamm Bowel Dis 19: 194-204.

11. Trifan A, Stanciu C, Stoica O, Girleanu I, Cojocariu C (2014) Impact of Clostridium difficile infection on inflammatory bowel disease outcome: A review. World J Gastroenterol 20: 11736-11742.

12. Razik R, Rumman A, Bahreini Z, McGeer A, Nguyen GC (2016) Recurrence of Clostridium difficile infection in patients with inflammatory bowel disease: The RECIDIVISM Study. Am J Gastroenterol 111: 1141-1146.

13. Vaishnavi C, Singh M, Kapoor P, Kochhar R (2015) Clinical and demographic profile of patients reporting for Clostridium difficile infection in a tertiary care hospital. Indian J Med Microbiol 33: 326-327.

14. Goodhand JR, Alazawi W, Rampton DS (2011) Systematic review: Clostridium difficile and inflammatory bowel disease. Aliment Pharmacol Ther 33: 428-441.

15. Nitzan O, Elias M, Chazan B, Raz R, Saliba W (2013) Clostridium difficile and inflammatory bowel disease: role in pathogenesis and implications in treatment. World J Gastroenterol 19: 7577-7585.

16. Clayton EM, Rea MC, Shanahan F, Quigley EM, Kiely B, et al. (2009) The vexed relationship between Clostridium difficile and inflammatory bowel disease: an assessment of carriage in an outpatient setting among patients in remission. Am J Gastroenterol 104: 1162-1169.

17. Hourigan SK, Chirumamilla SR, Ross T, Golub JE, Rabizadeh S, et al. (2013) Clostridium difficile carriage and serum antitoxin responses in children with inflammatory bowel disease. Inflamm Bowel Dis 19: 2744-2752.

18. Frank DN, Amand AL, Feldman RA, Boedeker EC, Harpaz N, et al. (2007) Molecular-phylogenetic characterization of microbial community imbalances in human inflammatory bowel diseases. Proc Natl Acad Sci USA 104: 13780-13785.

19. Sartor RB, Mazmanian SK (2012) Intestinal microbes in inflammatory bowel diseases. Am J Gastroenterol Suppl 1: 15-21.

20. Issa M, Vijayapal A, Graham MB, Beaulieu DB, Otterson MF, et al. (2007) Impact of Clostridium difficile on inflammatory bowel disease. Clin Gastroenterol Hepatol 5: 345-351.

21. Ben-Horin S, Margalit M, Bossuyt P, Maul J, Shapira Y, et al. (2009) Combination immunomodulator and antibiotic treatment in patients with inflammatory bowel disease and Clostridium difficile infection. Clin Gastroenterol Hepatol 7: 981-987.

22. Ott C, Girlich C, Klebl F, Plentz A, Iesalnieks, I, et al. (2011) Low risk of Clostridium difficile infections in hospitalized patients with inflammatory bowel disease in a German tertiary referral center. Digestion 84: 187-192.

23. Masclee GM, Penders J, Jonkers DM, Wolffs PF, Pierik MJ (2013) Is Clostridium difficile associated with relapse of inflammatory bowel disease? Results from a retrospective and prospective cohort study in The Netherlands. Inflamm Bowel Dis 19: 2125-2131.

24. Vaishnavi C, Singh M, Mahmood S, Kochhar R (2015) Prevalence and molecular types of Clostridium difficile isolates from faecal specimens of patients in a tertiary care centre. J. Med. Microbiol 64: 1297-1304. 
Citation: Vaishnavi C, Kochhar R (2017) Inflammatory Bowel Disease and Clostridium difficile Infection: A Report from a Tertiary Care Center of North India. J Gen Pract (Los Angel) 5: 310. doi:10.4172/2329-9126.1000310

Page 6 of 6

25. Cohen SH, Gerding DN, Johnson S, Kelly CP, Loo VG, et al. (2010) Clinical practice guidelines for Clostridium difficile infection in adults: 2010 update by the society for healthcare epidemiology of America (SHEA) and the infectious diseases society of America (IDSA). Infect Control Hosp Epidemiol 31: 431-455.

26. Vaishnavi C (2009) Established and potential risk factors for Clostridium difficile infection. Indian J Med. Microbiol 27: 291-302.

27. Libby DB, Bearman G (2009) Bacteremia due to Clostridium difficilereview of the literature. Int J Infect Dis 13: e305-e309.

28. Wultanska D, Banaszkiewicz A, Radzikowski A, Belkum AV (2010) Clostridium difficile infection in Polish pediatric outpatients with inflammatory bowel disease. Eur J Clin Microbiol Infect Dis 29: 1265-1270.

29. Kelsen JR, Kim J, Latta D, Smathers S, McGowan KL, et al. (2011) Recurrence rate of Clostridium difficile infection in hospitalized pediatric patients with inflammatory bowel disease. Inflamm Bowel Dis 17: 50-55.

30. Monaghan TM, Cockayne A, Mahida YR (2015) Pathogenesis of Clostridium difficile infection and its potential role in inflammatory bowel disease. Inflamm Bowel Dis 21: 1957-1966.

31. Bossuyt P, Verhaegen J, Assche GV, Rutgeerts P, Vermeire S (2009) Increasing incidence of Clostridium difficile-associated diarrhea in inflammatory bowel disease. J Crohns Colitis 3: 4-7.
32. Ananthakrishnan AN, McGinley EL, Saeian K, Binion DG (2011) Temporal trends in disease outcomes related to Clostridium difficile infection in patients with inflammatory bowel disease. Inflamm Bowel Dis 17: 976-983.

33. Navaneethan U, Mukewar S, Venkatesh PG, Lopez R, Shen B (2012) Clostridium difficile infection is associated with worse long term outcome in patients with ulcerative colitis. J Crohns Colitis 6: 330-336.

34. Nguyen GC, Kaplan GG, Harris ML, Brant SR (2008) A national survey of the prevalence and impact of Clostridium difficile infection among hospitalized inflammatory bowel disease patients. Am J Gastroenterol 103: 1443-1450.

35. Musa S, Thomson S, Cowan M, Rahman T (2010) Clostridium difficile infection and inflammatory bowel disease. Scand J Gastroenterol 45: 261-272.

36. Hughes M, Qazi T, Berg A, Weinberg, J, Chen, X, et al. (2016) Host immune response to Clostridium difficile infection in inflammatory bowel disease patients. Inflamm Bowel Dis 22: 853-861.

37. Ananthakrishnan AN, Issa M, Binion DG (2010) Clostridium difficile and inflammatory bowel disease. Med Clin North Am 94: 135-153.

38. Kucharzika T, Maaserb C (2014) Infections and chronic inflammatory bowel disease. Gastrointestinal Med Surg 30: 326-332. 